\title{
Experimental Evolution
}

\author{
Tadeusz J. Kawecki ${ }^{1,}{ }^{*}$, Richard E. Lenski ${ }^{2}$, Dieter Ebert ${ }^{3}$, Brian Hollis ${ }^{4}$, \\ Isabelle Olivieri ${ }^{5}$, Michael C. Whitlock ${ }^{6}$
}

Trends in Ecology and Evolution, 27: 547-560 (2012)

Except for a few changes made in proofs, the content of this preprint is identical with the published version of the paper

${ }^{1}$ Department of Ecology and Evolution, University of Lausanne, Switzerland, Email tadeusz.kawecki@unil.ch

${ }^{2}$ BEACON Center for the Study of Evolution in Action, Michigan State University, East Lansing, Michigan 48824, USA, Email lenski@msu.edu

${ }^{3}$ University of Basel, Zoological Institute, Vesalgasse 1, 4051 Basel, Switzerland, Email dieter.ebert@unibas.ch

${ }^{4}$ Department of Ecology and Evolution, University of Lausanne, Switzerland, Email brian.hollis@unil.ch

${ }^{5}$ Université Montpelier 2, CNRS, Institut des Sciences de l'Evolution, UMR 5554, 34095 Montpelier cedex 05, France, Email isabelle.olivieri@univ-montp2.fr

${ }^{6}$ Department of Zoology, University of British Columbia, Vancouver, Canada V6T 1Z4, Email whitlock@zoology.ubc.ca

\section{*Corresponding author}

\begin{abstract}
Experimental evolution is the study of evolutionary processes occurring in experimental populations in response to conditions imposed by the experimenter. This research approach is increasingly used to study adaptation, estimate evolutionary parameters and test diverse evolutionary hypotheses. Long applied in vaccine development, experimental evolution also finds new applications in biotechnology. Recent technological developments provide a path towards detailed understanding of the genomic and molecular basis of experimental evolutionary change, while new findings raise new questions that can be addressed with this approach. However, experimental evolution has important limitations, and the interpretation of results is subject to caveats resulting from small population sizes, limited timescale, the simplified nature of laboratory environments and, in some cases, the potential to misinterpret the selective forces and other processes at work.
\end{abstract}




\section{Experimental evolution as a research tool}

Evolutionary theories are usually inspired and tested by studying patterns - of phylogeny, of divergence between species or populations, of variation within populations, of genome structure and sequence - all of which reflect past evolution. Experimental evolution is an alternative research framework that offers the opportunity to study evolutionary processes experimentally in real time. The past decade has seen the fast growth of studies that tap into this potential, fuelled both by an increasing awareness of the power of this approach and by technological advances that facilitate analysis of the genetic and molecular basis of experimental evolution.

We define experimental evolution as the study of evolutionary changes occurring in experimental populations as a consequence of conditions (environmental, demographic, genetic, social, and so forth) imposed by the experimenter (Fig. 1 ). Thus, we do not consider cases of evolution in action that do not result from a planned and designed experiment. The above definition also excludes artificial selection (c.f. [1]), where breeding individuals are chosen explicitly by the investigator based on phenotypic values of defined traits or genotypes (e.g., at specific marker loci), thus enforcing a predetermined relationship between those traits or genotypes and fitness. By contrast, in experimental evolution selection can act on any and all traits and genes relevant to fitness under the environmental regimes of interest. Experimental evolution is sometimes called "laboratory natural selection"; however, some experimental evolution studies have been conducted in the field [2-4] and, moreover, some others have explicitly focused on other evolutionary forces including mutation, genetic drift, and gene flow [e.g., 5, 6]. Indeed, these other forces almost invariably act along with selection during experimental evolution, just as they do in nature.

Here, we provide an introduction to experimental evolution as a research approach, illustrating its power and versatility, but also highlighting its limitations and caveats. We discuss major aspects of study systems and experimental design, and we summarize recent technological advances that are revolutionizing the study of the genetic and molecular basis of experimental evolutionary change.

\section{Applications}

Experimental evolution has been employed to address diverse questions in many areas of evolutionary biology. Below we discuss several major types of questions, keeping in mind that different questions are often addressed in a single experiment. We also address the advantages of long-term experiments and some practical applications of experimental evolution.

Adaptation to specific environments. Many evolution experiments seek to understand how populations adapt to particular environmental conditions, usually defined in terms of a particular factor, such as temperature [7], nutrition [8], other environmental stressors [9], parasites [3], or competition $[10,11]$. A few of these studies are specifically designed to test hypothetical links between particular polymorphisms and fitness: they start from a gene pool constructed to be polymorphic at the focal locus or loci and then measure the response in terms of changes in allele frequency (e.g., $[12,13])$. Most studies, by contrast, rely on natural (i.e., uncontrolled) genetic variation sampled from a base population or generated de novo by random mutations. Even though these studies are often motivated by specific hypotheses about traits presumed to be relevant for adaptation (inspired, e.g., by patterns of inter-population variation in nature), other traits may evolve and provide additional and unexpected insights. Therefore, what one can learn from experimental 
evolution is relatively unconstrained by preconceptions about what traits and evolutionary processes are most important. The traditional focus on phenotypic aspects of adaptation has been increasingly combined with genomic data, facilitated by technological advances (Box 1).

Study of evolutionary trade-offs and constraints. It is widely assumed that many or most adaptations are associated with trade-offs, such that changes in traits that increase fitness in some environments or situations are deleterious in some other environments or situations. Experimental evolution provides ample evidence for widespread (though not universal) trade-offs in general and insights into their mechanisms in specific cases (e.g., [14]); the evidence has been reviewed elsewhere [15, 16]. As one example, experimental populations of Drosophila melanogaster that evolved postponed aging showed a decline in their early fecundity relative to populations that were allowed to breed immediately after emerging as adults [17]. That experiment and several similar ones were pivotal in the broader acceptance of an evolutionary explanation for aging $[18,19]$. Experimental evolution has also been employed to study constraints imposed by a lack of standing genetic variation for specific adaptation [20] and to address the notion that certain adaptations are unattainable by mutation. In this last category, evolution experiments have falsified the hypotheses that bacteria cannot evolve resistance to amphipatic antimicrobial peptides [21] and that E. coli cannot evolve to feed on citrate under oxic conditions [22]. The study of citrate use also throws light on the nature of the constraint: the appearance of the crucial mutation was contingent on earlier evolutionary changes. This contingency explains why this new function only evolved after 31,000 generations of experimental evolution and only in one of 12 replicate populations [22].

Estimating population genetic parameters. Mutation accumulation experiments, in which very small and initially isogenic populations evolve under conditions designed to minimize selection, are one important source of information about the statistical properties of spontaneous mutations affecting fitness and other quantitative traits. These statistics include the rate at which such mutations occur per genome, the distribution of their effects, the way they interact within (dominance) and between (epistasis) loci, and the variance and covariance they contribute to phenotypic variation per generation (reviewed in [6]). Laboratory adaptation experiments with bacteria, coupled with new population-genetic theory, have been devised to estimate the rates and effect-sizes of beneficial mutations [23-25]. In particular, one can estimate these parameters by following the dynamics of a neutral genetic marker seeded into a set of asexual populations, even without identifying the beneficial mutations themselves. Also, by constructing isogenic strains with specific combinations of evolved mutations, the extent and form of epistatic interactions among the beneficial mutations can be measured $[26,27]$. Experimental evolution has also been employed to estimate genetic variance in fitness within populations [28] as well as between replicate evolving lines [29, 30]. Finally, selection coefficients acting on alleles can sometimes be directly estimated from their dynamics under the experimental conditions $[29,31]$.

Testing evolutionary theories. The versatility of experimental evolution as a research framework is apparent in its applications to test predictions from evolutionary theory. It has been employed, for example, to address controversies as to whether particular evolutionary processes, postulated on theoretical grounds, are plausible. Such "proof of principle" studies have demonstrated, for example, that bacteria can evolve a new phenotypic switch (bet hedging) [32], that natural selection may favor male traits that directly reduce the fitness of their mates [33], and that some degree of reproductive isolation can evolve as a byproduct of divergent natural selection in different environments [34-36] or as a consequence of selection against hybrids [37, 38]. Conversely, no unequivocal evidence for 
founder-effect speciation has emerged from several experiments designed to test this model (e.g., $[5,39]$ and references therein); while these failed attempts do not prove that the process cannot occur, they do suggest that it is rare or requires more time to yield a discernible signal than the experiments allowed.

More often, experimental evolution has been employed to test specific predictions concerning the effect of general properties of the environment (e.g., spatial or temporal variability), demography (e.g., population size or structure, extrinsic mortality patterns, transmission rate and mode for parasites), social factors (e.g., relatedness) or other attributes of the population (e.g., mode of reproduction or mating system) on evolutionary processes and outcomes. Some of those hypotheses are listed in Table 1. We emphasize that this is not a comprehensive list and the studies cited are examples, chosen to cover the broad array of research topics to which experimental evolution has contributed. A comprehensive review of evidence for each hypothesis is beyond the scope of this paper.

Long-term experiments. Most evolution experiments start with one specific aim in mind, but as observations accrue new questions arise. A long-term experiment with $E$. coli-now past 55,000 generations - provides a case in point. The initial focus was on the dynamics of adaptation and divergence in 12 replicate populations, with mean fitness in the selection environment being the response of interest $[29,30]$. In time, analyses expanded to examine parallelism from morphological [29] to genetic levels [40, 41]; correlated responses, pleiotropy and specialization [42-44]; the evolution of mutation rates [45, 46]; forces maintaining diversity [47-49]; historical contingency [50] and the origin of a new function [22]; evolvability [30] and epistasis [26]; and the coupling between genomic and phenotypic evolution [51]. Long-term evolution experiments in Drosophila-some of which have been running for over 600 generations-have also yielded important insight into reversals of correlated evolutionary responses [52], the causes of aging and late-age mortality plateaus [53], and the relative importance of standing versus mutational variance in adaptation [54]. One of the longest running ecological experiments (started in 1856), which was designed to study the effects of fertilization and soil $\mathrm{pH}$ on plant community and ecosystem processes, led to insights on local adaptation and the evolution of reproductive isolation [55, 56]. Sufficiently long experiments might also probe the limits of adaptive evolution, at least for simple environments. Many evolution experiments show declining rates of phenotypic change, but does adaptive evolution eventually cease in the absence of environmental change?

Experimental evolution in medicine and technology. For decades, experimental evolution has been the method of choice for the development of live attenuated vaccines against viral and bacterial diseases, such as polio, tuberculosis, yellow fever, measles, mumps and rubella. For this purpose, the pathogens were serially passaged in other host species or artificial media, until their pathogenic effects in humans had attenuated as a correlated response to selection for improved growth in the new environment $[57,58]$. Thus, experimental evolution has contributed to saving millions of human lives, beginning even before it was understood that this method of vaccine production involves Darwinian evolution.

More recently, experimental evolution combined with genome sequencing and genetic mapping has been used to identify mutations that confer drug resistance to pathogens, before such mutations appear in nature (e.g. [59]). This approach might facilitate the rapid diagnosis of resistant infections if 
they appear in patients, allowing appropriate public-health measures including the development in advance of new drugs that target the resistant mutants.

Experimental evolution also has great potential in other areas of biotechnology. Serial passage of a pathogen on a particular host often leads to increased specialization and higher virulence on that host [57], and this principle has been used to produce more virulent strains of microbial [60] and metazoan [61] agents of biological pest control. Experimental evolution has also been used to produce biocatalysts and biocontrol agents with other desired properties, e.g., high thermal tolerance [62]. For some microbial systems this process can be fully automated [60,62]. From an engineering perspective, experimental evolution will undoubtedly be employed as a "bottom-up" complement or supplement to "top-down" genetic engineering methods to generate organisms for the production of biofuels [63] and for carbon sequestration.

Experimental evolution can even be extended to artificial living systems including some that are based on molecular processes and others that are computational in nature (Box 2). These artificial systems are being used not only to test basic hypotheses but also to evolve useful new products, from protein catalysts to software and even robots [64-66].

\section{Designing evolution experiments}

Study system. Many questions in evolutionary biology apply to a broad range of organisms or even to all. The choice of the study system thus becomes largely a matter of convenience. As a result, most evolutionary experiments have used one of several favorites, in particular E. coli, Pseudomonas, yeast and Drosophila (Table 1). Several phage-bacteria systems and Daphnia with its pathogens have been widely used to address questions about coevolution (Box 3 ). The relative paucity of evolution experiments on vascular plants [67-69] reflects in part their long generation times (even for Arabidopsis this is 2 months, compared to 2 weeks in Drosophila or Daphnia, 3-4 days in C. elegans, and hours in microbes). Concentrating on model systems has obvious advantages, such as integration of results from different fields and the availability of genomic information and tools. However, even if the questions an experimenter asks are general in nature, the answers obtained might nevertheless be specific to the taxon under study. Microbes differ from multicellular eukaryotes in many fundamental ways (Box 4), and so extrapolating between these domains must be done with care and may sometimes be problematic. Even closely related species may differ in ways relevant for evolution. For example, populations of Drosophila melanogaster are often polymorphic for large chromosomal inversions, which effectively suppress recombination over large regions of the genome; but such inversion polymorphisms are rare in the closely related $D$. simulans [70]. Thus, the evolution of the former species is more likely to be affected by linkage disequilibrium, which may produce more pronounced correlated responses in the absence of pleiotropy. Over time, the differences between general principles and idiosyncratic features of particular systems should emerge if the community of researchers uses a wide range of study systems.

More generally, the common model systems may also be more similar to one another-and unrepresentative of nature-precisely in the ways which make them so easy to study. These species have been chosen because they have short generation times. They all tolerate human-influenced environments, in some cases (e.g., Drosophila melanogaster) because they are human commensals. Some of them have recently increased in population size and adapted to the human-modulated 
environment, perhaps selecting for higher recombination rates and mutation rates than those in their sister taxa. Compared to their wild counterparts, the strains used in these studies have often already adapted to some laboratory conditions. They may prefer more constant abiotic conditions (e.g. temperature), use a narrower spectrum of resources that require little effort to locate, undergo little or no dispersal, have little need to react to stresses, have reduced capacity to interact with other species, and so forth. Whether these differences between the typical organisms used in experimental evolution and those more broadly representative of nature are important may depend on a particular study's goals. For example, experimental evolution is often well suited to asking whether some particular process or factor (e.g., population size) can be important in evolution, but this approach may not be appropriate to extrapolating parameter estimates (e.g., selection coefficients) to nature without appropriate caveats.

Regimes and controls. Hypothesis testing in evolution experiments typically involves comparisons between sets of populations evolving under different regimes, but originally derived from the same base population or the same ancestral genotype (Fig. 1c,d). Such comparisons quantify the differences in evolutionary response under the various regimes. Sometimes, a distinction can be made between "selection" (or "treatment") and "control" (or "unselected") regimes, perhaps suggesting that the "control" conditions mimic the ancestral conditions to which the base population or ancestral strain was adapted before the start of the experiment (e.g., [8, 71]). However, this assumption is hardly ever fulfilled, because the vast majority of any lineage's evolutionary history occurred outside the laboratory. In any case, the contribution of the different regimes to the observed divergence can be best evaluated if the ancestral population is included in the comparison. The phenotype of evolved populations can be compared in contemporaneous assays to the ancestral population if the latter can be preserved alive but prevented from evolving (Fig. 1a,b), e.g., by freezing or in resting stages such as seeds. At the genetic level, once candidate polymorphisms that may contribute to phenotypic evolution have been identified, allele frequencies in the various evolved populations can be compared with the ancestral population if a sample of genetic material for the latter is available (even if the ancestral organisms are no longer viable). Also, it should be kept in mind that a difference between evolved and ancestral populations might reflect greater inbreeding of the former (for sexually reproducing organisms: see below) or adaptation to aspects of the selection regime other than the factor being tested.

Experimental replicates. Isolated populations derived from the same gene pool will diverge with time even if they are maintained under the same environmental conditions. Such divergence will be driven by random genetic drift affecting pre-existing polymorphisms and the establishment of new mutations, by the order in which mutations appear, and by any uncontrolled environmental variation that affects the direction and intensity of selection. Divergence generated by these stochastic mechanisms can be further amplified by selection if the resulting differences in genetic background influence the fitness effects of alleles [72]. Therefore, genetic divergence between populations cannot be attributed with confidence to different regimes unless this divergence is shown to be greater than occurs in the absence of the imposed differences in regime. Rather, divergence between experimental regimes should be tested relative to variation among independently evolving replicate populations subjected to the same regime. In other words, experimental populations are the units of replication for testing evolutionary hypotheses.

Base population or ancestral genotype. Replicate populations are usually derived from a single base population or ancestral strain. In other cases, however, experimental populations may be paired or 
blocked based on their origin, before evolving under different experimental regimes. Evolutionary change is contingent on the initial gene pool, so starting from different ancestors may reduce the statistical power (because the different starting populations may respond differently); on the other hand, having multiple starting populations may increase the generality of any conclusions.

Evolution experiments that rely on pre-existing genetic variation (i.e., most non-microbial studies) start with a base population. That base population was itself typically founded with some dozens to thousands of individuals sampled from nature, then allowed to adapt to the laboratory environment for many generations while being maintained at a large size (e.g. [71, 73]). Such base populations will likely harbor more polymorphisms, including rare alleles, than typical laboratory stocks. An experimenter could also mix individuals from different natural populations or laboratory stocks to increase genetic variation, but doing so would generate linkage disequilibrium, which might be problematic depending on the question of interest.

Experimental population size and number of generations. While evolving microbial populations are usually maintained at sizes of millions, experimental populations in non-microbial systems are limited by practical considerations to thousands, hundreds or even dozens of breeding individuals. Small population sizes have important consequences for several aspects of evolution (Box 5). Of the studies cited in Table 1, many that started from outbred populations have detected divergence in mean trait values or fitness within 10-20, and sometimes as few as 3-8 generations (e.g., [68, 74, 75]). However, experiments that fail to produce an evolutionary response are often not published, and so these numbers should be viewed as optimistic. In microbial experiments, responses may occur within a single day (5-10 generations) when using strong selective agents, such as viruses and antibiotics. With more subtle selection for improved competitive ability, 200 or more generations might be needed before the first beneficial mutations rise to fixation [30]. Experiments designed to detect changes in variance [30,76], or to observe second-order effects on such traits as mutation rate [45], typically require more generations.

Controlling for maternal effects. Different experimental evolution regimes often involve different environmental, demographic, or social conditions. Conditions experienced by the parents often affect the phenotypes of their offspring (or even grand-offspring) via non-genetic maternal and paternal effects. Such effects can be mediated by egg or seed provisioning, signaling molecules in the cytoplasm, chromatin modification, and other epigenetic mechanisms [77]. Most evolution experiments focus on genetically-based changes. In order to eliminate effects caused by different parental environments, samples of populations from all regimes (and the revived ancestor where applicable) should be reared in a common environment for one or more generations before their divergence is assessed. The choice of this common parental environment can be complicated, however, if there is an interaction between genotypic and maternal-environment effects [78].

Controlling for differential inbreeding. Even if populations under different regimes are maintained at the same census size, the regime with stronger selection will have smaller effective population size [79], leading to a greater degree of inbreeding in sexually reproducing organisms. Greater inbreeding could, in turn, lead to a reduction in fitness components (inbreeding depression), which might be misinterpreted as a correlated response to selection (reflecting pleiotropy or linkage disequilibrium). Crossing replicate populations within selection regimes should restore heterozygosity and thus largely eliminate the inbreeding depression. If crosses between replicate populations within regimes exhibit the same pattern of phenotypic differences between regimes as the original populations, 
then the differences can be more safely interpreted as resulting from selection (e.g., [8]). However, such crosses may also show complex patterns if the phenotypically similar responses of replicate populations reflect different genetic mechanisms that interact in a non-additive way (e.g., [80]).

\section{Caveats and limitations}

Timescale and serendipity. Numerous success stories notwithstanding, experimental evolution has some limitations as a research approach, and the conclusions from evolutionary experiments are subject to caveats. Although experimental evolution can be extremely fast, some evolutionary processes may be too slow to be seen within the span of a research grant or even a researcher's professional lifetime. Limited insight from experimental evolution into speciation is a case in point; while a measurable degree of reproductive isolation has evolved in several experiments, it has never reached the degree of isolation expected between biological species [72] (except for the special mechanism of speciation via polyploidization of hybrids in plants [81]). Other processes, such as the origin of morphological novelty, may depend on rare sequences of mutational events or improbable outcomes of drift, such that the likelihood of them happening in an experiment is too low to justify the undertaking, especially if other approaches can provide empirical support. Still other questions have been difficult to address with experimental evolution for want of an appropriate study system. In particular, despite some progress (e.g., $[82,83])$, efforts to test hypotheses about the short-term advantages of sex have been hampered by the fact that, in species capable of both modes of reproduction, sexual and asexual offspring are usually physiologically or ecologically distinct.

Technical difficulties and laboratory artifacts. Evolution experiments can be compromised by contamination, i.e., inadvertent introduction of "immigrants" into experimental populations [84]. Other unexpected factors may confound the intended regimes. For example, in a study aimed to test the effect of extrinsic host mortality on parasite virulence, the host mortality regime became unexpectedly confounded with multiplicity of infection, completely altering the selective forces on the parasite [85]. Finally, populations may evolve to obviate the intended regimes; e.g., a study concerning the effect of ploidy on adaptation in yeast was thwarted when the initially haploid and tetraploid populations all evolved diploidy [86].

In studies aimed at understanding adaptation to a particular environmental factor, the results may depend on the way in which that factor is implemented. For example, selection for acute starvation resistance in Drosophila led to reduced locomotor activity [87]. This behavior was adaptive under the laboratory regime, where flies were deprived of food for a certain time and the survivors were given food later, because reduced locomotion conserves energy. However, food shortages in nature may often favor increased mobility to find new food sources. Indeed, as a plastic (phenotypic) response, flies become highly active when deprived of food [87]. Laboratory environments often confine mobile animals to small space, changing the context of social and sexual interactions; e.g., in contrast to nature, female Drosophila cannot escape aggressive sexual interactions, which inflates mating frequency and may amplify sexual conflict $[88,89]$. Such considerations indicate the need for caution in extrapolating particular adaptive outcomes from the laboratory to the field.

Population genetics of laboratory evolution. The population genetics of laboratory evolution may differ in important ways from evolution in nature. One reason is the small effective population size in experiments relative to nature, which has manifold consequences for evolution (Box 5). Also, in experiments with outbred populations, evolutionary responses will depend largely on standing 
genetic variation present in the base population, at least over the first 100 or so generations [90]. From a population genetic view, such experiments mimic evolution following abrupt environmental changes. The genetics of such responses are expected to differ from evolution that depends on new mutations in several ways; in particular, adaptations to abrupt changes are more likely to involve recessive alleles and alleles with smaller effects (reviewed in [91]).

Finally, owing to the simple environments and strong selection, laboratory evolution may involve alleles with different patterns of pleiotropy from those typical in nature. Many fitness-related traits are presumably affected by many alleles with diverse pleiotropic effects. In nature selection will usually act simultaneously on many aspects of the organism's phenotypes; hence, selection on any particular function or trait will often be weak. Thus, adaptation in nature is more likely to involve alleles that show few or no adverse pleiotropic effects (if such alleles exist). In contrast, experiments often impose strong selection on a single focal factor. Other sources of selection (e.g., suboptimal conditions, pathogens, locomotion and so on) are often absent or minimized. As a consequence, pleiotropic effects that would be deleterious in nature may be neutral or nearly so in the laboratory; as a case in point, about $60 \%$ of single-gene deletions in yeast are effectively neutral under optimal laboratory conditions [92]. Furthermore, the availability of alleles with small or no antagonistic pleiotropic effects may be limited by the small sizes of laboratory populations. Laboratory selection may thus more often involve alleles with strong adverse pleiotropic effects than evolution in nature. Therefore, experimental evolution studies may tend to overstate the importance of evolutionary trade-offs.

\section{Experimental evolution in the field}

Some of the concerns discussed above can be circumvented by performing evolution experiments in natural environments. A pioneering evolution experiment in the field was initiated in 1976 by transferring a guppy (Poecilia reticulata) population between environments with different predation regimes, leading to seminal insights into the evolution of life histories, sexual signaling, mate preferences, and predator-prey coevolution [4, 93-95]. Despite this early and successful start, there have been few experimental evolution studies in the field. Such studies involve moving populations, manipulating natural environments, or both, and these actions impose logistical challenges and may also raise legal, ethical, or conservation issues. Another difficulty is the need to confine experimental populations, which limits the approach to island-like habitats [2, 3, 96-98]. Finally, the environment as a whole is not controlled, making the experiments more likely to fail if, e.g., populations become locally extinct. Many of the hypotheses in Table 1 concern general demographic, genetic, social or other factors, and their predictions are unrelated to a specific environment and its complexity, so it can be argued that field tests in such cases are not worth the additional effort. On the other hand, for reasons discussed in the preceding section, where the question concerns adaptation to specific environmental factors, laboratory environments may introduce artifacts. Furthermore, populations in the field can be much larger. Some experiments are impractical in the laboratory; e.g., by introducing predators to islands, Losos et al. [96] showed that predation drives the evolution of an arboreal lifestyle in Anolis lizards. Finally, field experiments allow one to study the direct and indirect effects of evolutionary change on ecosystem processes [94].

Mesocosms (e.g., artificial ponds) offer an intermediate between laboratory and field studies. Experiments performed in parallel in natural habitats and in mesocosms produced reassuringly 
similar effects of predation on the evolution of color pattern evolution in guppies [93] and on the advantage of immigrant alleles in genetically depauperate populations of Daphnia [2].

Finally, in some cases one can verify the relevance of laboratory-evolved adaptations to fitness in nature by assaying experimentally derived organisms under field conditions. For example, Drosophila from populations selected for cold tolerance were more likely than flies from control populations to be recaptured at food sources hours after their release into the field in cold weather, but not at mild temperatures [99]. This finding indicates that the experimental adaptation to cold under laboratory conditions translated into improved ability to survive and find food under cold conditions in the field.

\section{Conclusions}

The potential value of experimental evolution as a research approach has long been recognizedalready in 1892 a book titled Experimental Evolution proposed using this methodology to resolve the controversy between the Darwinian and Lamarckian theories of evolution [100]. The past decade has seen increasing application of experimental evolution to an expanding range of questions, while advances in genomic technology are beginning to provide unprecedented insights into the genetic and molecular bases of evolutionary change. These and other technological advances open new avenues, while discoveries in fields including genetics, developmental biology, and global change pose new questions that can be tackled with experimental evolution (see Box 6, "New Opportunities, New Challenges").

Experimental evolution also offers a unique opportunity to improve science education. While paleontology and comparative studies provide ample evidence for evolution, the fact that scientists can observe evolution in action through manipulative experiments is an eye-opener to people for whom "seeing is believing". Also, many organisms used for research in experimental evolution can be readily deployed in teaching laboratories. For example, a class can quickly evolve bacteria to resist antibiotics [101]. If time permits, students could then compete the evolved and ancestral strains in the absence of antibiotic to test for tradeoffs. Using Drosophila, students can, over a semester, observe selection against alleles that are readily scored, such as those that disrupt wing morphology [102]. (Of course, such experiments in classrooms require suitable facilities and appropriately trained teachers, and they must comply with institutional policies and local regulations on biological experiments.) Using digital organisms-computer programs that self-replicate, mutate and compete in a virtual world-students can watch evolution before their eyes as they vary environments and other factors and observe their effects on the emergence of new phenotypes [103]. As the use of experimental evolution continues to expand in the research community, we hope it will also have a growing impact on science education.

\section{Acknowledgements}

TJK and DE are supported by the Swiss National Science Foundation. REL's research is supported by the US National Science Foundation (DEB-1019989 and Cooperative Agreement DBI-0939454). IO is supported by CNRS and Institut Universitaire de France; MCW by Natural Sciences and Engineering Research Council (Canada). We thank two anonymous reviewers for comments, and D. J. Kemp, M. Travisano, J. Radwan, and G. J. Velicer for sharing photographs. 


\section{Box 1. Genomics and experimental evolution}

The first complete genome sequence was for the phage $\Phi \times 174$, and its 5,375-bp sequence appeared in 1982. A draft of the $3,000-\mathrm{Mb}$ human genome was published in 2001. These achievements were remarkable in their day but now, thanks to technical advances, whole-genome re-sequencing is accessible for experimental evolution studies. In 1997 Bull et al. [104] sequenced nine DX174 isolates that had evolved on two hosts. In 2007 Velicer and colleagues [105] sequenced the genome of a Myxococcus xanthus derivative that had evolved from socially cooperative to cheating and back to cooperative. In 2009 Barrick and Lenski [49] deeply sequenced seven whole-population samples that spanned 40,000 generations from an evolving Escherichia coli population to find genetic polymorphisms. In 2010 genomics was extended to experimentally evolved eukaryotes, Saccharomyces cerevisiae [106] and Drosophila melanogaster [54]. The application of genomics to experimental evolution may soon be limited only by the imagination of the investigator and the quality of the study design. Other high-throughput approaches are also increasingly useful for experimental evolution including characterizing an organism's capacity to use diverse resources (e.g., [43]) as well as proteomic (e.g., [107]), transcriptional (e.g., [40]) and metabolic profiling (e.g., [108]).

To date, studies at the interface of genomics and experimental evolution have ranged from descriptive ones that demonstrate new technologies $[109,110]$ or find genes of interest $[105,106$, 111] to quantitative analyses of diverse conceptual issues. How repeatable is evolution at the levels of nucleotides, genes and pathways $[27,51,104,112,113]$ ? How do epistatic interactions and the order of mutations affect evolvability, marginal fitness effects and the origin of new functions [26, 27, 113-115]? What are genomic mutation rates and the spectrum of mutational types, and how do they evolve $[49,51,116-118]$ ? What are the dynamics of genome evolution in relation to phenotypic change and in terms of hard versus soft selective sweeps [49, 51, 54, 104, 111, 113]?

While these high-throughput methods provide new opportunities, they can also be difficult for analysis and interpretation. In particular, demonstrating causal links between specific changes at the genomic or transcriptional level with divergence in morphology, physiology, behavior or life history remains challenging, especially in non-microbial systems. These methods often identify divergence in allele frequencies at hundreds of loci [54, 111] or in expression of hundreds of transcripts [119]. Owing to linkage, drift, and statistical false-positives, not all of these differences will have been caused by adaptation. Therefore, such data must be interpreted with caution. 


\section{Box 2. Experimental evolution with artificial Life}

One of the goals of experimental evolution, as a field, is to test general hypotheses about evolutionary processes-in contrast to many comparative studies that seek to understand the evolution of a particular trait in a given phylogenetic context. Yet, all of life on Earth derives from the same primordial ancestors, so how general can evolutionary tests be? As Maynard Smith put it [120]: "So far, we have been able to study only one evolving system, and we cannot wait for interstellar flight to provide us with a second. If we want to discover generalizations about evolving systems, we will have to look at artificial ones."

Artificial evolving systems include synthetic replicators built from organic molecules [121,122] and digital organisms living in virtual worlds [123, 124]. As Dennett says [125]: "The process of natural selection is substrate-neutral ... evolution will occur whenever and wherever three conditions are met: replication, variation (mutation), and differential fitness (competition)." The Avida system is a computational platform developed for this research, one in which digital organisms are programs that replicate, mutate and compete [124, 126]. (Both research and educational versions of Avida are freely available on the web.) The organisms can manipulate bit-strings and, if they perform an operation appropriate to their environment, they obtain additional energy to run their genetic programs. Starting from a simple ancestor that can replicate but not perform other functions, populations can evolve a complex computational metabolism [127]. As in nature, selection acts on the phenotypes of digital organisms, not on their genetic encoding.

Digital systems offer short generations, controllable environments and automated analyses including, for example, lines of descent (showing every intermediate from the ancestor to an evolved state of interest) and genotype-phenotype maps (showing the effect of mutating each genomic position on every phenotype). To date, experiments with digital organisms have addressed diverse issues including the origin of parasites [123], effect of mutation rate on robustness [128], historical contingency [129], origin of complex functions [127], ontogeny and phylogeny [130], evolution of sex [131], role of pleiotropy in ecological specialization [132], recovery from extinctions [133] and selection for altruism [134]. One can even extend this approach to embodied robots by evolving their morphology, behavior, or both in a virtual world constrained by physical laws, then building the computationally evolved robots [64]. In this way, robots can evolve to pursue or evade other robots as predators or prey [135], cooperate [136], and communicate with one another [137, 138]. 


\section{Box 3. Experimental coevolution}

The term "coevolution" refers to two phenomena: (1) the evolution of interacting species whereby evolutionary change in one species induces evolutionary change in another species and vice versa; and (2) a similar process occurring between genes and traits of the same population but expressed in different classes of individuals (e.g., sexes, mother-offspring) or with different modes of transmission (e.g., selfish genetic elements and their suppressors). Most evolution experiments have concentrated on antagonistic coevolution, where the fitness of both parties cannot be simultaneously maximized. Rather than being externally controlled, a crucial aspect of the environment in coevolutionary experiments is the coevolving party and thus a moving target.

Disentangling co-from evolution. Identifying evolved changes that result from the coevolutionary feedback often requires comparing coevolutionary regimes (where the interacting species evolve together) with "unilateral" regimes where only one species is allowed to evolve and the other is kept static [139-141]. An analogous approach to coevolution between the sexes is challenging because the sexes share a gene pool, but inroads have been made using sophisticated breeding designs [142].

Dynamics of coevolution. Antagonistic coevolution proceeds by two fundamentally different modes [143]. (1) Time-lagged negatively frequency-dependent selection favors phenotypes that were rare or absent a few generations ago. This mode often leads to unstable dynamics, such as an arms-race. (2) Selective sweep coevolution occurs when beneficial mutations arise and spread to fixation. Coevolution experiments have been performed to investigate by which mode coevolution proceeds and to test how coevolutionary dynamics are affected by, and in turn influence, genetic and demographic factors $[139,144,145]$. Systems in which samples of coevolving populations can be preserved and revived allow time-shift-experiments [146]. These experiments involve reciprocal transplants in time, where populations of one antagonist (e.g., host) sampled at time $t_{1}$ are confronted with the other antagonist (e.g., parasite) sampled at times $t_{0}$ (past), $t_{1}$ (contemporary) and $t_{2}$ (future). Parasites from the future are expected to be more infective to hosts from time $t_{1}$ under a selective sweep model, but not under negative frequency-dependent selection models [147, 148].

Coevolution-driven divergence. In a coevolving system, stochastic changes in one antagonist may change selection on the other antagonist, and the resulting evolutionary change may feed back on the evolution of the first species and so on. Coevolution will thus magnify stochastic effects and accelerate divergence between isolated populations, as seen in a several experiments with phage and bacteria [27, 139, 149]. 
Box 4. Microbes versus macrobes

Microbes were largely ignored by evolutionary biologists for many decades. Eventually, though, the utility of microbes for experimental evolution became clear. Their obvious advantages include short generations, large populations, and the ease with which environments can be controlled and manipulated. Another important feature is that most microbes can be stored frozen in a nonevolving state and later revived (this is also possible for some animals and plant seeds). This property effectively enables travel in time: the experimenter can directly compare organisms from different generations, e.g., by competing derived and ancestral genotypes to measure their relative fitness [30, 150]. Indeed, by performing simultaneous assays with organisms from many different generations, one minimizes the effects of uncontrolled fluctuations in conditions that might confound interpretation of data collected at different times. One can also perform "replays" where evolution is restarted from intermediate generations to test whether an outcome of interest, such as the origin of a new function, was contingent on earlier changes $[22,27,113]$.

Evolutionary experiments with microbes and other organisms differ in several ways, the importance of which may depend on the question of interest. Most experiments with microbes start with a single clone and depend on new mutations to generate variation. Evolution often occurs by consecutive selective sweeps, although frequency-dependent interactions and clonal interference (competition between beneficial mutations) also can be important [48, 151-153]. In contrast, experimental evolution in non-microbial experiments is mostly fuelled by genetic variation already present in the initial population, and alleles are regularly recombined by sex. As a consequence, microbial populations may evolve more slowly, at least on a generational basis. Furthermore, some phenomena central to the evolution of many plants and animals, such as sex, sexual selection, parental care and speciation are either absent or involve very different mechanisms in microbes (particularly bacteria or viruses), limiting the utility of the latter as model systems for those phenomena in the former. On the other hand, eukaryotic microbes have been used to study the evolution of reproductive isolation [36, 37] and sexual selection [154]. Furthermore, factors such as mutation rates [46], recombination [83, 155-157] and genetic relatedness [158-162] can be manipulated in some microbes to examine their evolutionary effects.

Finally, microbes are less familiar than the larger organisms we see all around us. As a consequence, most evolutionary biologists have better intuition about what phenotypic traits and environmental factors matter for animals and plants - for example, beak size and seed hardness - than for the physiological traits and physicochemical factors that determine the fitness of microbes. The potential for microbes to exhibit complex life histories [161, 163, 164] and social behaviors [160, 161, 165] has only recently become appreciated. Thus, microbes are now being used as model systems to study the evolution of traits that biologists traditionally ascribed only to multi-cellular organisms. 
Box 5. Effects of population size in experimental evolution

By necessity, experimental populations are orders of magnitude smaller than in nature. Therefore, many fewer alleles are available to respond to selection, for two reasons. First, some alleles are lost due to drift in small populations, with the loss rate inversely proportional to the effective population size $\left(N_{\mathrm{e}}\right)$. Effective sizes for eukaryotes are usually thought to be up to an order of magnitude smaller than census sizes $(N)$ [166]; factors that reduce $N_{\mathrm{e}}$ compared to $N$ include selection at other loci, random variation in reproductive output, biased sex ratio and fluctuations in population sizes. In microbial populations propagated by serial transfer (e.g. [30, 167]), the effective size depends strongly on the transfer size, not the maximum population size [30,168]. For diploid organisms, the loss of genetic diversity can also lead to inbreeding depression, which lowers fitness in subsequent generations. Deleterious alleles can fix by random drift if the population is small enough ( $N_{e}$ less than the reciprocal of the selection coefficient); in fact mutation-accumulation experiments rely on this process [6]. The loss of diversity and inbreeding are unlikely to matter too much over the first 10 generations or so, provided the effective population size is a few dozen or more.

Second, the number of new mutations per generation is proportional to the number of genomes in the population, i.e., $N$ or $2 N$ depending on ploidy. The relative importance of standing variation and new mutation depends on the size of the population and the length of the experiment. Hill [169] estimates that in long-term experimental selection studies, many fixed alleles arise as new mutations, and of course many experimental populations are started from a genetically uniform stock, making new mutations all-important. Responses to selection can be significantly limited by population size $[46,167,170,171]$.

Small population sizes can affect the progress of evolution in a number of ways. For multi-cellular sexual organisms, the rate of adaptation in a laboratory population is likely to be mutation limited after it exhausts (through selection or drift) the starting genetic variation. Such species typically have long generation times, and few alleles have time to reach fixation during a typical experiment. On the other hand, it may be possible to have much larger populations of single-celled organisms. Indeed, in some cases all one-step point mutations are estimated to have arisen multiple times [22]. In such cases, however, the rate of adaptive evolution still may be limited by small population sizes because some adaptations may require two or more mutations, and the order in which they occur and their epistatic interactions may constrain evolution [22, 26, 113]. Simultaneous double mutations are very rare, and exploring the space of all possible double mutations would require very large populations. 
Box 6. New Opportunities, New Challenges

Automation. Experimental evolution requires a substantial investment of time and labor to maintain the populations under their intended regimes, while the return on this investment - in terms of results and publications - takes months, years, or even decades and is uncertain. The problem may be alleviated to some extent by progress in automation of population maintenance under particular regimes (e.g., www.ksepdx.com/live_transferring.htm and [62]) and of assays of physiological [43], morphological [172], and life history phenotypes [173].

Use of transgenics to verify genetic basis of adaptation. Advances in genetic manipulation techniques offer increasing possibilities to examine the causal links between genomic changes, phenotypes, and fitness. For example, specific point mutations can now be introduced or recombined in several model systems [174, 175]. In Drosophila melanogaster, the GAL4-UAS dual technique is now routinely used to express any exogenous transcript in specific tissues or cells [176] or, in combination with RNA interference techniques, to down-regulate endogenous transcripts [177]. Such techniques permit independent tests of the phenotypic and fitness effects of particular mutations or changes in gene expression observed in the course of experimental evolution.

Very long projects. Some ecological experiments (e.g., [55]) and artificial selection projects (e.g., [178]) have now been running for over a century, spanning multiple generations of researchers. Comparable efforts in experimental evolution (already proposed in 1892 [100]) would offer insights into rare events and slow processes, such as the origin of morphological novelties, the functional differentiation of duplicated genes, and perhaps even the speciation process taken to completion.

Epigenetic inheritance. The past decade provided evidence that some quasi-hereditary information can be encoded in patterns of chromatin modification (e.g., DNA methylation). Evidence for such epigenetic inheritance is pervasive in plants [179], but has also been reported to affect longevity in Caenorhabditis elegans [180] as well as wing development and possibly reproductive mode in aphids [181]. Epigenetic inheritance is only beginning to be incorporated into evolutionary theory [182] and experimental evolution may contribute to this development.

Protein-coding or regulatory bases of adaptation. Experimental evolution can contribute new data about the relative contributions of mutations in protein-coding versus regulatory regions to adaptive evolution, at least those changes occurring between closely related taxa [183, 184].

Experimental evolution and anthropogenic change. There is growing awareness that evolutionary processes can sometimes be rapid enough to have implications for conservation of species and ecosystems [185-187]. Experimental evolution can contribute to understanding of such processes as species invasions [188] and evolutionary rescue from local extinction [189]. Experimental evolution studies may also influence the scenarios of biotic responses to global change such as evolutionary responses of algae to elevated $\mathrm{CO}_{2}$ levels [190]. 


\section{References}

1 Garland, T. and Rose, M.R., eds (2009) Experimental Evolution. University of California Press

2 Ebert, D., et al. (2002) A selective advantage to immigrant genes in a Daphnia metapopulation. Science $295,485-488$

3 Zbinden, M., et al. (2008) Experimental evolution of field populations of Daphnia magna in response to parasite treatment. J. Evol. Biol. 21, 1068-1078

4 Reznick, D.A., et al. (1990) Experimentally induced life-history evolution in a natural population. Nature 346, 357-359

5 Rundle, H.D. (2003) Divergent environments and population bottlenecks fail to generate premating isolation in Drosophila pseudoobscura. Evolution 57, 2557-2565

6 Halligan, D.L. and Keightley, P.D. (2009) Spontaneous mutation accumulation studies in evolutionary genetics. In Annu. Rev. Ecol. Evol. Syst., pp. 151-172

7 Bennett, A.F. and Lenski, R.E. (1993) Evolutionary adaptation to temperature. II. Thermal niches of experimental lines of Escherichia coli. Evolution 47, 1-12

8 Kolss, M., et al. (2009) Life history consequences of adaptation to larval nutritional stress in Drosophila. Evolution 63, 2389-2401

9 Dhar, R., et al. (2011) Adaptation of Saccharomyces cerevisiae to saline stress through laboratory evolution. J. Evol. Biol. 24, 1135-1153

10 terHorst, C.P. (2011) Experimental evolution of protozoan traits in response to interspecific competition. J. Evol. Biol. 24, 36-46

11 Santos, M., et al. (1997) Density-dependent natural selection in Drosophila: evolution of growth rate and body size. Evolution 51, 420-432

12 Fitzpatrick, M.J., et al. (2007) Maintaining a behaviour polymorphism by frequency-dependent selection on a single gene. Nature 447, 210-212

13 Murray, R.L. and Cutter, A.D. (2011) Experimental evolution of sperm count in protandrous selffertilizing hermaphrodites. J. Exp. Biol. 214, 1740-1747

14 Lenski, R.E. (1988) Experimental studies of pleiotropy and epistasis in Escherichia coli. I. Variation in competitive fitness among mutants resistant to virus T4. Evolution 42, 425-432

15 Fry, J.D. (2003) Detecting ecological trade-offs using selection experiments. Ecology 84, 1672-1678

16 Roff, D.A. and Fairbairn, D.J. (2007) The evolution of trade-offs: where are we? J. Evol. Biol. 20, 433-447

17 Rose, M.R. (1984) Laboratory evolution of postponed senescence in Drosophila melanogaster. Evolution 38, 1004-1010

18 Williams, G.C. (1957) Pleiotropy, natural selection and the evolution of senescence. Evolution 11, 398-411

19 Hamilton, W.D. (1966) The moulding of senescence by natural selection. J. Theor. Biol. 12, 12-45

20 Hoffmann, A.A., et al. (2003) Low potential for climatic stress adaptation in a rainforest Drosophila species. Science 301, 100-102

21 Perron, G.G., et al. (2006) Experimental evolution of resistance to an antimicrobial peptide. Proceedings of the Royal Society B-Biological Sciences 273, 251-256

22 Blount, Z.D., et al. (2008) Historical contingency and the evolution of a key innovation in an experimental population of Escherichia coli. Proc. Natl. Acad. Sci. USA 105, 7899-7906

23 Hegreness, M., et al. (2006) An equivalence principle for the incorporation of favorable mutations in asexual populations. Science 311, 1615-1617 
24 Perfeito, L., et al. (2007) Adaptive mutations in bacteria: High rate and small effects. Science 317, 813-815

25 Rozen, D.E., et al. (2002) Fitness effects of fixed beneficial mutations in microbial populations. Current Biology 12, 1040-1045

26 Khan, A.l., et al. (2011) Negative epistasis between beneficial mutations in an evolving bacterial population. Science 332, 1193-1196

27 Meyer, J.R., et al. (2012) Repeatability and contingency in the evolution of a key innovation in phage Lambda. Science 335, 428-432

28 Fowler, K., et al. (1997) Genetic variation for total fitness in Drosophila melanogaster. Proc. R. Soc. Lond. B 264, 191-199

29 Lenski, R.E. and Travisano, M. (1994) Dynamics of adaptation and diversification - a 10,000generation experiment wiht bacterial populations. Proc. Natl. Acad. Sci. USA 91, 6808-6814

30 Lenski, R.E., et al. (1991) Long-term experimental evolution in Escherichia coli. I. Adaptation and divergence during 2,000 generations. Am. Nat. 138, 1315-1341

$31 \mathrm{Hollis}$, B., et al. (2009) Sexual selection accelerates the elimination of a deleterious mutant in Drosophila melanogaster. Evolution 63, 324-333

32 Beaumont, H.J.E., et al. (2009) Experimental evolution of bet hedging. Nature 462, 90-U97

33 Rice, W.R. (1996) Sexually antagonistic male adaptation triggered by experimental arrest of female evolution. Nature 381, 232-234

34 Rundle, H.D., et al. (2005) Divergent selection and the evolution of signal traits and mating preferences. Plos Biology 3, 1988-1995

35 Dodd, D.M.B. (1989) Reproductive isolation as a consequence of adaptive divergence in Drosophila pseudoobscura. Evolution 43, 1308-1311

36 Dettman, J.R., et al. (2008) Divergent adaptation promotes reproductive isolation among experimental populations of the filamentous fungus Neurospora. Bmc Evolutionary Biology 8

37 Leu, J.Y. and Murray, A.W. (2006) Experimental evolution of mating discrimination in budding yeast. Current Biology 16, 280-286

38 Matute, D.R. (2010) Reinforcement can overcome gene flow during speciation in Drosophila. Current Biology 20, 2229-2233

39 Moya, A., et al. (1995) Founder effect speciation theory: failure of experimental corroboration. Proc. Natl. Acad. Sci. USA 92, 3983-3986

40 Cooper, T.F., et al. (2003) Parallel changes in qene expression after 20,000 generations of evolution in Escherichia coli. Proc. Natl. Acad. Sci. USA 100, 1072-1077

41 Woods, R., et al. (2006) Tests of parallel molecular evolution in a long-term experiment with Escherichia coli. Proc. Natl. Acad. Sci. USA 103, 9107-9112

42 Travisano, M. and Lenski, R.E. (1996) Long-term experimental evolution in Escherichia coli .4. Targets of selection and the specificity of adaptation. Genetics 143, 15-26

43 Cooper, V.S. and Lenski, R.E. (2000) The population genetics of ecological specialization in evolving Escherichia coli populations. Nature 407, 736-739

44 Meyer, J.R., et al. (2010) Parallel changes in host resistance to viral infection during 45,000 generations of relaxed selection. Evolution 64, 3024-3034

45 Sniegowski, P.D., et al. (1997) Evolution of high mutation rates in experimental populations of $E$. coli. Nature 387, 703-705

46 de Visser, J., et al. (1999) Diminishing returns from mutation supply rate in asexual populations. Science $283,404-406$ 
47 Elena, S.F. and Lenski, R.E. (1997) Long-term experimental evolution in Escherichia coli.7. Mechanisms maintaining genetic variability within populations. Evolution 51, 1058-1067

48 Rozen, D.E., et al. (2009) Death and cannibalism in a seasonal environment facilitate bacterial coexistence. Ecol. Lett. 12, 34-44

49 Barrick, J.E. and Lenski, R.E. (2009) Genome-wide mutational diversity in an evolving population of Escherichia coli. Cold Spring Harbor Symposia on Quantitative Biology 74

50 Travisano, M., et al. (1995) Experimental tests of the roles of adaptation, chance, and history in evolution. Science 267, 87-90

51 Barrick, J.E., et al. (2009) Genome evolution and adaptation in a long-term experiment with Escherichia coli. Nature 461, 1243-U1274

52 Archer, M.A., et al. (2003) Breakdown in correlations during laboratory evolution. II. Selection on stress resistance in Drosophila populations. Evolution 57, 536-543

53 Rose, M.R., et al. (2002) Evolution of late-life mortality in Drosophila melanogaster. Evolution 56, 1982-1991

54 Burke, M.K., et al. (2010) Genome-wide analysis of a long-term evolution experiment with Drosophila. Nature 467, 587-U111

55 Silvertown, J., et al. (2006) The Park Grass Experiment 1856-2006: Its contribution to ecology. J. Ecol. 94, 801-814

56 Silvertown, J., et al. (2005) Reinforcement of reproductive isolation between adjacent populations in the Park Grass Experiment. Heredity 95, 198-205

57 Ebert, D. (1998) Experimental evolution of parasites. Science 282, 1432-1435

58 Plotkin, S.A. and Plotkin, S.L. (2011) The development of vaccines: how the past led to the future. Nature Reviews Microbiology 9, 889-893

59 Hunt, P., et al. (2010) Experimental evolution, genetic analysis and genome re-sequencing reveal the mutation conferring artemisinin resistance in an isogenic lineage of malaria parasites. BmC Genomics 11

60 Kolodny-Hirsch, D.M. and VanBeek, N.A.M. (1997) Selection of a morphological variant of Autographa californica nuclear polyhedrosis virus with increased virulence following serial passage in Plutella xylostella. J. Invertebr. Pathol. 69, 205-211

61 Dion, E., et al. (2011) Rapid evolution of parasitoids when faced with the symbiont-mediated resistance of their hosts. J. Evol. Biol. 24, 741-750

62 de Crecy, E., et al. (2009) Directed evolution of a filamentous fungus for thermotolerance. BMC Biotechnol. 9

63 Arnold, F.H. (2008) The race for new biofuels. Engineering \& Science 2, 12-19

64 Lipson, H. and Pollack, J.B. (2000) Automatic design and manufacture of robotic lifeforms. Nature 406, 974-978

65 Brustad, E.M. and Arnold, F.H. (2011) Optimizing non-natural protein function with directed evolution. Curr. Opin. Chem. Biol. 15, 201-210

66 Koza, J.R. (1992) Genetic Programming: On the Programming of Computers by Means of Natural Selection. MIT Press

67 Goldringer, I., et al. (2006) Rapid differentiation of experimental populations of wheat for heading time in response to local climatic conditions. Annals of Botany 98, 805-817

68 Dorken, M.E. and Pannell, J.R. (2009) Hermaphroditic sex allocation evolves when mating opportunities change. Current Biology 19, 514-517 
69 Roels, S.A.B. and Kelly, J.K. (2011) Rapid evolution caused by pollinator loss in Mimulus guttatus. Evolution 65, 2541-2552

70 Capy, P. and Gibert, P. (2004) Drosophila melanogaster, Drosophila simulans: so similar yet so different. Genetica 120, 5-16

71 Magalhaes, S., et al. (2007) Adaptation in a spider mite population after long-term evolution on a single host plant. J. Evol. Biol. 20, 2016-2027

72 Coyne, J.A. and Orr, H.A. (2004) Speciation. Sinauer Associates

73 Rice, W.R., et al. (2005) Inter-locus antagonistic coevolution as an engine of speciation: Assessment with hemiclonal analysis. Proc. Natl. Acad. Sci. USA 102, 6527-6534

74 Koskella, B. and Lively, C.M. (2009) Evidence for negative frequency-dependent selection during experimental coevolution of a freshwater snail and a sterilizing trematode. Evolution 63, 22132221

75 Porcher, E., et al. (2006) Genetic differentiation of neutral markers and quantitative traits in predominantly selfing metapopulations: confronting theory and experiments with Arabidopsis thaliana. Genet. Res. Camb. 87, 1-12

76 Yeaman, S., et al. (2010) No effect of environmental heterogeneity on the maintenance of genetic variation in wing shape in Drosophila melanogaster. Evolution 64, 3398-3408

77 Mousseau, T.A., et al. (2009) Evolution of maternal effects: past and present. Philos. Trans. R. Soc. B-Biol. Sci. 364, 1035-1038

78 Magalhaes, S., et al. (2011) Environmental effects on the detection of adaptation. J. Evol. Biol. 24, 2653-2662

79 Santiago, E. and Caballero, A. (1998) Effective size and polymorphism of linked neutral loci in populations under directional selection. Genetics 149, 2105-2117

80 Kawecki, T.J. and Mery, F. (2006) Genetically idiosyncratic responses of Drosophila melanogaster populations to selection for improved learning ability. J. Evol. Biol. 19, 1265-1274

81 Soltis, P.S. and Soltis, D.E. (2009) The role of hybridization in plant speciation. In Annual Review of Plant Biology, pp. 561-588, Annual Reviews

82 Becks, L. and Agrawal, A.F. (2010) Higher rates of sex evolve in spatially heterogeneous environments. Nature 468, 89-92

83 Goddard, M.R., et al. (2005) Sex increases the efficacy of natural selection in experimental yeast populations. Nature $434,636-640$

84 Houle, D., et al. (1994) The genomic mutation rate for fitness in Drosophila (vol 359, pg 58, 1992). Nature 371, 358-358

85 Ebert, D. and Mangin, K.L. (1997) The influence of host demography on the evolution of virulence of a microsporidian gut parasite. Evolution 51, 1828-1837

86 Gerstein, A.C., et al. (2006) Genomic convergence toward diploidy in Saccharomyces cerevisiae. Plos Genetics 2, 1396-1401

87 Williams, A.E., et al. (2004) The respiratory pattern in Drosophila melanogaster selected for desiccation resistance is not associated with the observed evolution of decreased locomotory activity. Physiol. Biochem. Zool. 77, 10-17

88 Stewart, A.D., et al. (2005) Assessing putative interlocus sexual conflict in Drosophila melanogaster using experimental evolution. Proceedings of the Royal Society B-Biological Sciences $272,2029-2035$

89 Gromko, M.H. and Markow, T.A. (1993) Courtship and remating in field populations of Drosophila. Anim. Behav. 45, 253-262 
90 Hermisson, J. and Pennings, P.S. (2005) Soft sweeps: molecular population genetics of adaptation from standing genetic variation. Genetics 169, 2335-2352

91 Barrett, R.D.H. and Schluter, D. (2008) Adaptation from standing genetic variation. Trends Ecol. Evol. 23, 38-44

92 Hillenmeyer, M.E., et al. (2008) The chemical genomic portrait of yeast: Uncovering a phenotype for all genes. Science 320, 362-365

93 Endler, J.A. (1980) Natural selection on color patterns in Poecilia reticulata. Evolution 34, 76-91

94 Palkovacs, E.P., et al. (2009) Experimental evaluation of evolution and coevolution as agents of ecosystem change in Trinidadian streams. Philos. Trans. R. Soc. B-Biol. Sci. 364, 1617-1628

95 Reznick, D.N., et al. (1997) Evaluation of the rate of evolution in natural populations of guppies (Poecilia reticulata). Science 275, 1934-1937

96 Losos, J.B., et al. (2004) Predator-induced behaviour shifts and natural selection in fieldexperimental lizard populations. Nature 432, 505-508

97 Losos, J.B., et al. (1997) Adaptive differentiation following experimental island colonization in Anolis lizards. Nature 387, 70-73

98 Barrett, R.D.H., et al. (2011) Rapid evolution of cold tolerance in stickleback. Proceedings of the Royal Society B-Biological Sciences 278, 233-238

99 Kristensen, T.N., et al. (2007) Can artificially selected phenotypes influence a component of field fitness? Thermal selection and fly performance under thermal extremes. Proceedings of the Royal Society B-Biological Sciences 274, 771-778

100 de Varigny, H. (1892) Experimental Evolution. MacMillan

101 Krist, A.C. and Showsh, S.A. (2007) Experimental evolution of antibiotic resistance in bacteria. American Biology Teacher 69, 94-97

102 Plunkett, A.D. and Yampolsky, L.Y. (2010) When a fly has to fly to reproduce: selection against conditional recessive lethals in Drosophila. American Biology Teacher 72, 12-15

103 Speth, E.B., et al. (2009) Using Avida-ED for teaching and learning about evolution in undergraduate introductory biology courses. Evolution: Education and Outreach 2, 415-428

104 Bull, J.J., et al. (1997) Exceptional convergent evolution in a virus. Genetics 147, 1497-1507

105 Velicer, G.J., et al. (2006) Comprehensive mutation identification in an evolved bacterial cooperator and its cheating ancestor. Proc. Natl. Acad. Sci. USA 103, 8107-8112

106 Araya, C.L., et al. (2010) Whole-genome sequencing of a laboratory-evolved yeast strain. Bmc Genomics 11

107 Knight, C.G., et al. (2006) Unraveling adaptive evolution: how a single point mutation affects the protein coregulation network. Nat. Genet. 38, 1015-1022

108 Ibarra, R.U., et al. (2002) Escherichia coli K-12 undergoes adaptive evolution to achieve in silico predicted optimal growth. Nature 420, 186-189

109 Albert, T.J., et al. (2005) Mutation discovery in bacterial genomes: metronidazole resistance in Helicobacter pylori. Nature Methods 2, 951-953

110 Shendure, J., et al. (2005) Accurate multiplex polony sequencing of an evolved bacterial genome. Science 309, 1728-1732

111 Turner, T.L., et al. (2011) Population-based resequencing of experimentally evolved populations reveals the genetic basis of body size variation in Drosophila melanogaster. Plos Genetics 7

112 Herring, C.D., et al. (2006) Comparative genome sequencing of Escherichia coli allows observation of bacterial evolution on a laboratory timescale. Nat. Genet. 38, 1406-1412 
113 Woods, R.J., et al. (2011) Second-order selection for evolvability in a large Escherichia coli population. Science 331, 1433-1436

114 Lee, D.H. and Palsson, B.O. (2010) Adaptive evolution of Escherichia coli K-12 MG1655 during growth on a nonnative carbon cource, L-1,2-Propanediol (vol 76, pg 4158, 2010). Applied and Environmental Microbiology 76, 4158-4168

115 Chou, H.H., et al. (2011) Diminishing returns epistasis among beneficial mutations decelerates adaptation. Science 332, 1190-1192

116 Wielgoss, S., et al. (2011) Mutation rate inferred from synonymous substitutions in a long-term evolution experiment with Escherichia coli. G3: Genes, Genomes, Genetics 1, 183-186

117 Haag-Liautard, C., et al. (2007) Direct estimation of per nucleotide and genomic deleterious mutation rates in Drosophila. Nature 445, 82-85

118 Denver, D.R., et al. (2009) A genome-wide view of Caenorhabditis elegans base-substitution mutation processes. Proc. Natl. Acad. Sci. USA 106, 16310-16314

119 Sorensen, J.G., et al. (2007) Gene expression profile analysis of Drosophila melanogaster selected for resistance to environmental stressors. J. Evol. Biol. 20, 1624-1636

120 Maynard Smith, J. (1992) Byte-sized evolution. Nature 355, 772-773

121 Bartel, D.P. and Szostak, J.W. (1993) Isolation of new ribozymes from a large pool of random sequences. Science 261, 1411-1418

122 Joyce, G.F. (2009) Evolution in an RNA world. Cold Spring Harbor Symposia on Quantitative Biology 74, 17-23

123 Ray, T.S. (1992) An approach to the synthesis of life. In Artificial Life II (Langton, C.G., et al., eds), pp. 371-408, Springer

124 Adami, C. (1998) Introduction to Artificial Life. Springer

125 Dennett, D. (2002) The new replicators. In Encyclopedia of Evolution (Pagel, M.D., ed), pp. E83E92, Oxford University Press

126 Ofria, C. and Wilke, C.O. (2004) Avida: A software platform for research in computational evolutionary biology. Artificial Life 10, 191-229

127 Lenski, R.E., et al. (2003) The evolutionary origin of complex features. Nature 423, 139-144

128 Wilke, C.O., et al. (2001) Evolution of digital organisms at high mutation rates leads to survival of the flattest. Nature 412, 331-333

129 Yedid, G. and Bell, G. (2002) Macroevolution simulated with autonomously replicating computer programs. Nature 420, 810-812

130 Clune, J., et al. (2012) Ontogeny tends to recapitulate phylogeny in digital organisms. Am. Nat. in press.

131 Misevic, D., et al. (2006) Sexual reproduction reshapes the genetic architecture of digital organisms. Proceedings of the Royal Society B-Biological Sciences 273, 457-464

132 Ostrowski, E.A., et al. (2007) Ecological specialization and adaptive decay in digital organisms. Am. Nat. 169, E1-E20

133 Yedid, G., et al. (2009) Selective Press Extinctions, but Not Random Pulse Extinctions, Cause Delayed Ecological Recovery in Communities of Digital Organisms. Am. Nat. 173, E139-E154

134 Clune, J., et al. (2011) Selective pressures for accurate altruism targeting: evidence from digital evolution for difficult-to-test aspects of inclusive fitness theory. Proceedings of the Royal Society B-Biological Sciences 278, 666-674

135 Floreano, D. and Keller, L. (2010) Evolution of adaptive behaviour in robots by means of Darwinian selection. Plos Biology 8 
136 Waibel, M., et al. (2011) A quantitative test of Hamilton's rule for the evolution of altruism. Plos Biology 9

137 Floreano, D., et al. (2007) Evolutionary conditions for the emergence of communication in robots. Current Biology 17, 514-519

138 Mitri, S., et al. (2011) Relatedness influences signal reliability in evolving robots. Proceedings of the Royal Society B-Biological Sciences 278, 378-383

139 Buckling, A. and Rainey, P.B. (2002) The role of parasites in sympatric and allopatric host diversification. Nature 420, 496-499

140 Schulte, R.D., et al. (2010) Multiple reciprocal adaptations and rapid genetic change upon experimental coevolution of an animal host and its microbial parasite. Proc. Natl. Acad. Sci. USA $107,7359-7364$

141 Hollis, B. (2012) Rapid antagonistic coevolution between strains of the social amoeba Dictyostelium discoideum. Proc. R. Soc. Lond. B in press

142 Rice, W.R. (1998) Male fitness increases when females are eliminated from gene pool: Implications for the Y chromosome. Proc. Natl. Acad. Sci. USA 95, 6217-6221

143 Ebert, D. (2008) Host-parasite coevolution: Insights from the Daphnia-parasite model system. Current Opinion in Microbiology 11, 290-301

144 Vogwill, T., et al. (2011) Coevolving parasites enhance the diversity-decreasing effect of dispersal. Biology Letters 7, 578-580

145 Yoshida, T., et al. (2007) Cryptic population dynamics: Rapid evolution masks trophic interactions. Plos Biology 5, 1868-1879

146 Gaba, S. and Ebert, D. (2009) Time-shift experiments as a tool to study antagonistic coevolution. Trends Ecol. Evol. 24, 226-232

147 Decaestecker, E., et al. (2007) Host-parasite 'Red Queen' dynamics archived in pond sediment. Nature 450, 870-U816

148 Hall, A.R., et al. (2011) Host-parasite coevolutionary arms races give way to fluctuating selection. Ecol. Lett. 14, 635-642

149 Morgan, A.D., et al. (2005) The effect of migration on local adaptation in a coevolving hostparasite system. Nature 437, 253-256

150 Dykhuizen, D.E. and Hartl, D.L. (1983) Selection in chemostats. Microbiol. Rev. 47, 150-168

151 Gerrish, P.J. and Lenski, R.E. (1998) The fate of competing beneficial mutations in an asexual population. Genetica 102-3, 127-144

152 Treves, D.S., et al. (1998) Repeated evolution of an acetate-crossfeeding polymorphism in longterm populations of Escherichia coli. Molecular Biology and Evolution 15, 789-797

153 Rainey, P.B. and Travisano, M. (1998) Adaptive radiation in a heterogeneous environment. Nature 394, 69-72

154 Rogers, D.W. and Greig, D. (2009) Experimental evolution of a sexually selected display in yeast. Proceedings of the Royal Society B-Biological Sciences 276, 543-549

155 Malmberg, R.L. (1977) Evolution of epistasis and advantage of recombination in populations of bacteriophage T4. Genetics 86, 607-621

156 Cooper, T.F. (2007) Recombination speeds adaptation by reducing competition between beneficial mutations in populations of Escherichia coli. Plos Biology 5, 1899-1905

157 Schoustra, S., et al. (2010) Fitness-associated sexual reproduction in a filamentous fungus. Current Biology 20, 1350-1355

158 Turner, P.E. and Chao, L. (1999) Prisoner's dilemma in an RNA virus. Nature 398, 441-443 
159 Chao, L. and Levin, B.R. (1981) Structured habitats and the evolution of anticompetitor toxins in bacteria. Proc. Natl. Acad. Sci. USA 78, 6324-6328

160 Velicer, G.J., et al. (2000) Developmental cheating in the social bacterium Myxococcus xanthus. Nature 404, 598-560

161 Khare, A., et al. (2009) Cheater-resistance is not futile. Nature 461, 980-U240

162 Kaltz, O. and Bell, G. (2002) The ecology and genetics of fitness in Chlamydomonas. XII. Repeated sexual episodes increase rates of adaptation to novel environments. Evolution 56, 1743-1753

163 Ackermann, M., et al. (2003) Senescence in a bacterium with asymmetric division. Science 300, 1920-1920

164 Ratcliff, W.C., et al. (2012) Experimental evolution of multicellularity. Proc. Natl. Acad. Sci. USA $109,1595-1600$

165 Griffin, A.S., et al. (2004) Cooperation and competition in pathogenic bacteria. Nature 430, 10241027

166 Frankham, R. (1995) Effective population size adult population size ratios in wildlife - a review. Genet. Res. Camb. 66, 95-107

167 Samani, P. and Bell, G. (2010) Adaptation of experimental yeast populations to stressful conditions in relation to population size. J. Evol. Biol. 23, 791-796

168 Wahl, L.M. and Gerrish, P.J. (2001) The probability that beneficial mutations are lost in populations with periodic bottlenecks. Evolution 55, 2606-2610

169 Hill, W.G. (1982) Rates of change in quantitative traits from fixation of new mutations. Proceedings of the National Academy of Sciences of the United States of America-Biological Sciences 79, 142-145

170 Weber, K.E. (1990) Increased selection response in larger populations. I. Selection for wing-tip height in Drosophila melanogaster at three population sizes. Genetics 125, 579-584

171 Weber, K.E. and Diggins, L.T. (1990) Increased selection response in larger populations. II. Selection for ethanol vapor resistance in Drosophila melanogaster at two population sizes. Genetics 125, 585-597

172 Houle, D., et al. (2003) Automated measurement of Drosophila wings. Bmc Evolutionary Biology 3

173 Stearns, S.C., et al. (1987) A device for collecting flies of precisely determined post-hatching age. Drosophila Information Service 66, 167-179

174 Storici, F., et al. (2001) In vivo site-directed mutagenesis using oligonucleotides. Nat. Biotechnol. 19, 773-776

175 Wesolowska, N. and Rong, Y.K.S. (2010) The past, present and future of gene targeting in Drosophila. Fly 4, 53-59

176 Brand, A.H. and Perrimon, N. (1993) Targeted gene expression as a means of altering cell fates and generating dominant phenotypes. Development 118, 401-415

177 Dietzl, G., et al. (2007) A genome-wide transgenic RNAi library for conditional gene inactivation in Drosophila. Nature 448, 151-U151

178 Dudley, J.W. and Lambert, R.J. (2004) 100 generations of selection for oil and protein in corn. Plant Breeding Reviews 24, 79-110

179 Hauser, M.-T., et al. (2011) Transgenerational epigenetic inheritance in plants. Biochimica Et Biophysica Acta-Gene Regulatory Mechanisms 1809, 459-468

180 Greer, E.L., et al. (2011) Transgenerational epigenetic inheritance of longevity in Caenorhabditis elegans. Nature 479, 365-U204 
181 Walsh, T.K., et al. (2010) A functional DNA methylation system in the pea aphid, Acyrthosiphon pisum. Insect Molecular Biology 19, 215-228

182 Day, T. and Bonduriansky, R. (2011) A unified approach to the evolutionary consequences of genetic and nongenetic inheritance. Am. Nat. 178, E18-E36

183 Hoekstra, H.E. and Coyne, J.A. (2007) The locus of evolution: Evo devo and the genetics of adaptation. Evolution 61, 995-1016

184 Haag, E.S. and Lenski, R.E. (2011) L'enfant terrible at 30: the maturation of evolutionary developmental biology. Development 138, 2633-2637

185 Lankau, R., et al. (2011) Incorporating evolutionary principles into environmental management and policy. Evolutionary Applications 4, 315-325

186 Lavergne, S., et al. (2010) Biodiversity and climate change: integrating evolutionary and ecological responses of species and communities. In Annual Review of Ecology, Evolution, and Systematics, Vol 41 (Futuyma, D.J., et al., eds), pp. 321-350, Annual Reviews

187 Bell, G. and Collins, S. (2008) Adaptation, extinction and global change. Evolutionary Applications 1, 3-16

188 Lee, C.E., et al. (2007) Response to selection and evolvability of invasive populations. Genetica $129,179-192$

189 Bell, G. and Gonzalez, A. (2011) Adaptation and evolutionary rescue in metapopulations experiencing environmental deterioration. Science 332, 1327-1330

190 Collins, S., et al. (2006) Changes in C uptake in populations of Chlamydomonas reinhardtii selected at high CO2. Plant Cell Environ. 29, 1812-1819

191 Burch, C.L. and Chao, L. (1999) Evolution by small steps and rugged landscapes in the RNA virus \$6. Genetics 151, 921-927

192 Bull, J.J., et al. (2003) Experimental evolution yields hundreds of mutations in a functional viral genome. Journal of Molecular Evolution 57, 241-248

193 Whitlock, M.C., et al. (2002) Persistence of changes in the genetic covariance matrix after a bottleneck. Evolution 56, 1968-1975

194 Bryant, E.H. and Meffert, L.M. (1993) The effect of serial founder-flush cycles on quantitative genetic variation in the housefly. Heredity $70,122-129$

195 Wade, M.J., et al. (1996) Inbreeding: Its effect on response to selection for pupal weight and the heritable variance in fitness in the flour beetle, Tribolium castaneum. Evolution 50, 723-733

196 Swindell, W.R. and Bouzat, J.L. (2005) Modeling the adaptive potential of isolated populations: Experimental simulations using Drosophila. Evolution 59, 2159-2169

197 Reboud, X. and Bell, G. (1997) Experimental evolution in Chlamydomonas. III. Evolution of specialist and generalist types in environments that vary in space and time. Heredity 78, 507-514 198 Turner, P.E. and Elena, S.F. (2000) Cost of host radiation in an RNA virus. Genetics 156, 14651470

199 Mackay, T.F.C. (1980) Genetic variance, fitness, and homeostasis in varying environments: an experimental check of the theory. Evolution 34, 1219-1222

200 Snook, R.R., et al. (2005) Experimental manipulation of sexual selection and the evolution of courtship song in Drosophila pseudoobscura. Behav. Genet. 35, 245-255

$201 \mathrm{Kemp}$, D.J., et al. (2009) Predicting the direction of ornament evolution in Trinidadian guppies (Poecilia reticulata). Proceedings of the Royal Society B-Biological Sciences 276, 4335-4343

202 Fricke, C. and Arnqvist, G. (2007) Rapid adaptation to a novel host in a seed beetle (Callosobruchus maculatus): The role of sexual selection. Evolution 61, 440-454 
203 Promislow, D.E.L., et al. (1998) Adult fitness consequences of sexual selection in Drosophila melanogaster. Proc. Natl. Acad. Sci. USA 95, 10687-10692

204 Hollis, B. and Houle, D. (2011) Populations with elevated mutation load do not benefit from the operation of sexual selection. J. Evol. Biol. 24, 1918-1926

205 Rundle, H.D., et al. (2006) The roles of natural and sexual selection during adaptation to a novel environment. Evolution 60, 2218-2225

206 Tilszer, M., et al. (2006) Evolution under relaxed sexual conflict in the bulb mite Rhizoglyphus robini. Evolution 60, 1868-1873

207 Martin, O.Y. and Hosken, D.J. (2003) Costs and benefits of evolving under experimentally enforced polyandry or monogamy. Evolution 57, 2765-2772

208 Stearns, S.C., et al. (2000) Experimental evolution of aging, growth, and reproduction in fruitflies. Proc. Natl. Acad. Sci. USA 97, 3309-3313

209 Macke, E., et al. (2011) Experimental evolution of reduced sex ratio adjustment under local mate competition. Science 334, 1127-1129

210 Chao, L., et al. (1992) Muller's ratchet and the advantage of sex in the RNA virus $\phi 6$. Evolution 46, 289-299

211 Wade, M.J. (1980) An experimental study of kin selection. Evolution 34, 844-855

212 Kerr, B., et al. (2006) Local migration promotes competitive restraint in a host-pathogen 'tragedy of the commons'. Nature 442, 75-78

213 Manhes, P. and Velicer, G.J. (2011) Experimental evolution of selfish policing in social bacteria. Proc. Natl. Acad. Sci. USA 108, 8357-8362

214 Taylor, D.R., et al. (2002) Conflicting levels of selection in the accumulation of mitochondrial defects in Saccharomyces cerevisiae. Proc. Natl. Acad. Sci. USA 99, 3690-3694

215 Kuzdzal-Fick, J.J., et al. (2011) High relatedness is necessary and sufficient to maintain multicellularity in Dictyostelium. Science 334, 1548-1551

216 Mery, F. and Kawecki, T.J. (2004) The effect of learning on experimental evolution of resource preference in Drosophila melanogaster. Evolution 58, 757-767

217 Chao, L., et al. (1977) Complex community in a simple habitat - experimental study with bacteria and phage. Ecology 58, 369-378

218 Meyer, J.R., et al. (2006) Prey evolution on the time scale of predator-prey dynamics revealed by allele-specific quantitative PCR. Proc. Natl. Acad. Sci. USA 103, 10690-10695

219 Magalon, H., et al. (2010) Host growth conditions influence experimental evolution of life history and virulence of a parasite with vertical and horizontal transmission. Evolution 64, 2126-2138

220 Meffert, L.M. and Bryant, E.H. (1991) Mating propensity and courtship behavior in serially bottlenecked lines of the housefly. Evolution 45, 293-306

221 Ferea, T.L., et al. (1999) Systematic changes in gene expression patterns following adaptive evolution in yeast. Proc. Natl. Acad. Sci. USA 96, 9721-9726

222 Wichman, H.A., et al. (1999) Different trajectories of parallel evolution during viral adaptation. Science 285, 422-424

223 Velicer, G.J. and Yu, Y.T.N. (2003) Evolution of novel cooperative swarming in the bacterium Myxococcus xanthus. Nature 425, 75-78

224 Tomkins, J.L., et al. (2011) Habitat complexity drives experimental evolution of a conditionally expressed secondary sexual trait. Current Biology 21, 569-573 
Table 1. Examples of evolutionary hypotheses with references to selected studies that have tested (but not necessarily supported) those hypotheses using evolution experiments. " $F$ " indicates an evolution experiment in the field.

\section{Hypothesis}

Mutation and adaptation

Adaptation occurs mostly via many mutations of small effect

Fitness effects of beneficial mutations show negative epistatic interactions

Mutators (strains with elevated mutation rates) may evolve during adaptation to a novel environment

Mutators may enhance rates of adaptive evolution

\section{Genetic drift and inbreeding}

Genetic drift reshapes genetic variance-covariance matrices

Bottlenecks do not reduce and may increase additive genetic variance

Offspring of immigrants have high fitness in small inbred populations

\section{Environmental variability}

Spatial heterogeneity with restricted gene flow favors local adaptation in metapopulations

Spatial environmental heterogeneity drives adaptive radiation

Fluctuating environments favor generalist genotypes, and constant environments favor specialist genotypes

Fluctuating environments maintain genetic variation

\section{Sexual selection and conflict}

Intensity of sexual signals increases under strong sexual selection

Intensity of sexual signals increases when predation pressure is relaxed

Sexual selection facilitates elimination of deleterious alleles

Sexual selection leads to reduction (-) or increase $(+)$ in non-sexual fitness components
Organism and references

Bacteriophage $\varphi 6$ [191];

Escherichia coli [23-25]

Bacteriophage T7 [192];

Escherichia coli [26];

Methylobacterium extorquens [115]

Escherichia coli [45]

Escherichia coli [45]

Drosophila melanogaster [193]

Musca domestica [194]; Tribolium castaneum [195]; Drosophila melanogaster [196]

Daphnia magna (F) [2]

Arabidopsis thaliana [75]

Pseudomonas fluorescens [153]

Chlamydomonas reinhardtii [197] Vesicular stomatitis virus [198]; Escherichia coli [43]; digital organisms [132]

Drosophila melanogaster [76, 199]

Drosophila pseudoobscura [200]; Saccharomyces cerevisiae [154]

Poecilia reticulata (F) [201]

Drosophila melanogaster [31]

Callosobruchus maculatus $(+)$ [202]; Drosophila melanogaster $(+)$ [203], (-) [204]; D. serrata (-) [205] 
Polygamy favors male traits that reduce fitness of their mates (interlocus sexual conflict) Drosophila melanogaster [33]; Rhizoglyphus robini (mite) [206];

Sepsis cynipseaii (fly) [207]

\section{Life history and sex allocation}

High extrinsic mortality leads to the evolution of shorter intrinsic lifespan

High predation favors high reproductive effort

Antagonistic pleiotropy contributes to late-life mortality plateau

Sex allocation in hermaphrodites evolves towards the Fisherian ratio

Local mate completion favors female-biased sex ratio

Drosophila melanogaster [208]

Poecilia reticulata (F) [95]

Drosophila melanogaster [53]

Mercurialis annua (plant) [68]

Tetranychus urticae (mite) [209]

\section{Sexual reproduction and mating systems}

Fitness declines in asexual populations by Muller's ratchet

Sex and recombination accelerate adaptation to a novel environment

Bacteriophage $\varphi 6[210]$

Chlamydomonas reinhardtii [162];

Saccharomyces cerevisiae [83];

Escherichia coli [156]

Incidence of sex increases in heterogeneous environments (in species with facultative sex)

Self-fertilization evolves under pollinator limitation

Sexual reproduction favors altered gene interactions and modularity

Brachionus calyciflorus (rotifer) [82]

Mimulus guttatus (plant) [69]

Bacteriophage T4 [155];

digital organisms [131]

\section{Kin selection and cooperation}

Relatedness favors restraint from cannibalism

Tribolium confusum [211]

Limited migration and local extinction promotes competitive restraint

Bacteriophage T4 [212]

Cooperators evolve to suppress social cheaters

Myxococcus xanthus [213]

Parasitic mitochondria evolve when among-cell selection is weak

Saccharomyces cerevisiae [214]

Single-cell bottlenecks promote cooperation among cells in multicellular organisms

Conditions favoring large size may lead to evolution of multicellularity

Dictyostelium discoideum [215]

Saccharomyces cerevisiae [164]

\section{Behavior and cognition}

Variation in foraging behavior is maintained by negative frequency-dependent selection

Drosophila melanogaster [12]

Opportunity to learn may accelerate genetically-based adaptation (Baldwin effect)

Drosophila melanogaster [216]

\section{Host-parasite interactions}

Parasites or predators select for host or prey resistance, and resistance is costly

Daphnia magna and microsporidian parasite $(\mathrm{F})[2]$; Escherichia coli and various bacteriophages [14, 27, 44, 217]; 


\begin{tabular}{|c|c|}
\hline & $\begin{array}{l}\text { Chlorella vulgaris (algae) and } \\
\text { Brachionus calyciflorus (rotifer) } \\
{[218]}\end{array}$ \\
\hline Parasites impose negative frequency-dependent selection on the host & $\begin{array}{l}\text { Potamopyrgus antipodarum } \\
\text { (gastropode) and Microphallus sp } \\
\text { (trematode) [74] }\end{array}$ \\
\hline Host-parasite coevolution drives divergence and local adaptation & $\begin{array}{l}\text { Escherichia coli and bacteriophage } \\
\varphi 6[139,149]\end{array}$ \\
\hline $\begin{array}{l}\text { Vertical transmission and lower virulence evolve under conditions of high host } \\
\text { population growth }\end{array}$ & $\begin{array}{l}\text { Paramecium caudatum and } \\
\text { Holospora undulate [219] }\end{array}$ \\
\hline \multicolumn{2}{|l|}{ Speciation } \\
\hline Divergent selection leads to premating isolation & $\begin{array}{l}\text { Drosophila pseudoobscura [35]; D. } \\
\text { serrata [34] }\end{array}$ \\
\hline Divergent selection leads to postmating isolation & Neurospora sp. [36] \\
\hline Hybrid inferiority leads to reinforcement of prezygotic reproductive isolation & Drosophila yakuba [38] \\
\hline Repeated bottlenecks lead to reproductive isolation (not supported) & $\begin{array}{l}\text { Drosophila pseudoobscura [5, 39]; } \\
\text { Musca domestica [220] }\end{array}$ \\
\hline \multicolumn{2}{|l|}{ Repeatability of evolution } \\
\hline $\begin{array}{l}\text { Adaptation in independent populations occurs via parallel changes in gene expression, } \\
\text { parallel mutations, or parallel enrichment of pre-existing alleles }\end{array}$ & $\begin{array}{l}\text { Escherichia coli [40]; } \\
\text { Saccharomyces cerevisiae [221]; } \\
\text { Various bacteriophages [27, 104, } \\
\text { 222]; Drosophila melanogaster [54] }\end{array}$ \\
\hline Traits less correlated with fitness are more influenced by chance and history & Escherichia coli [50] \\
\hline Ontogeny recapitulates phylogeny & Digital organisms [127] \\
\hline
\end{tabular}



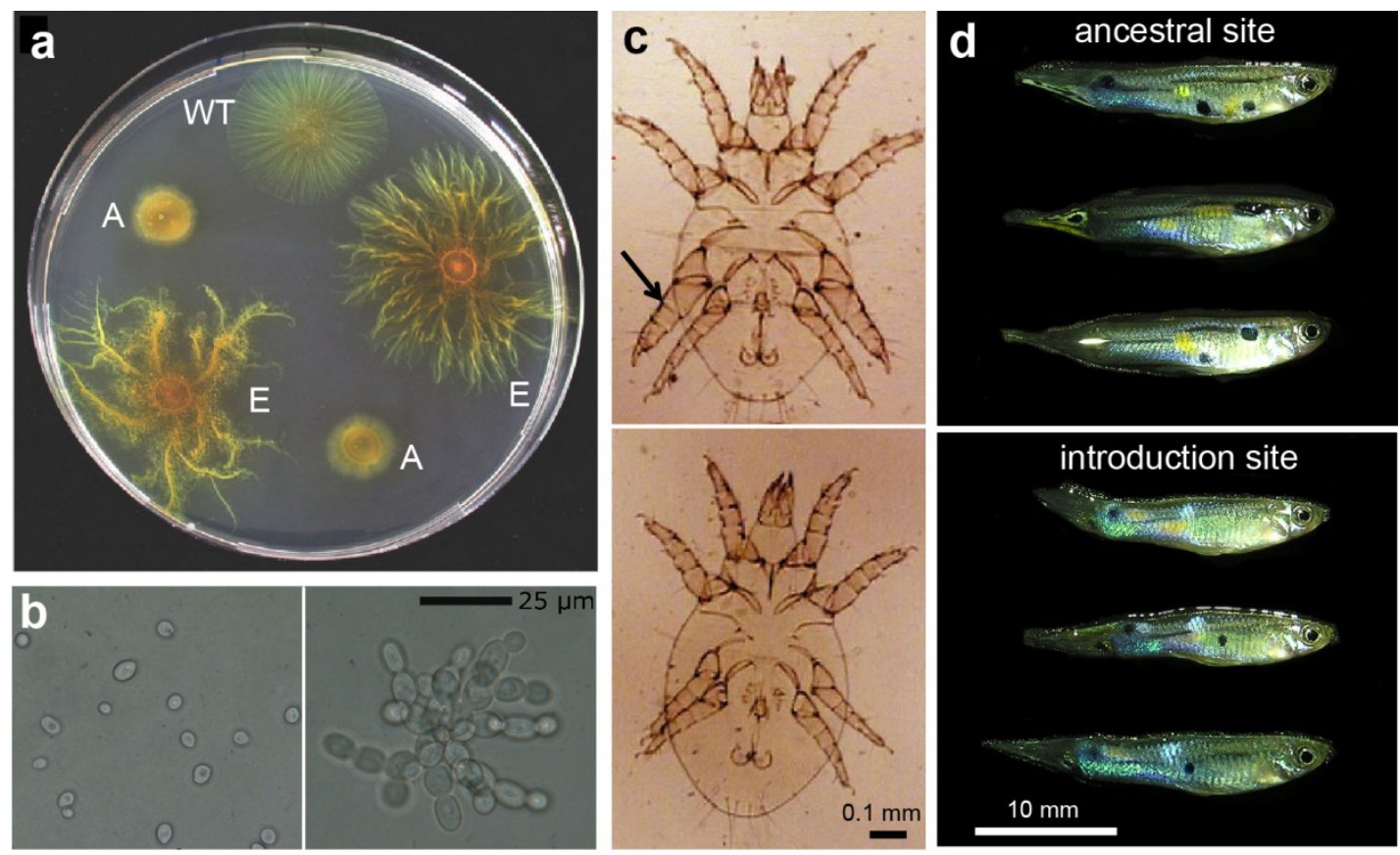

Figure 1. Examples of experimentally evolved phenotypic changes. (a) Cells of the social bacterium Myxococcus xanthus cooperate to swarm across solid surfaces in search of food and to form multicellular fruiting bodies. Strains initially unable to swarm due to loss of a necessary gene ("A") evolved alternative swarming mechanisms, leading to novel swarm morphologies ("E") that are markedly different from the ancestral form ("WT") [223]. Each swarm contains many millions of cells. Reprinted by permission from Macmillan Publishers Ltd: Nature 425: 75-78, copyright (2003). (b) Multicellular "snowflake" yeast (right) experimentally evolved from a single-celled ancestor (left) under conditions favoring large size (adapted from [164], copyright William C. Ratcliff et al. [164]). (c) Two male morphs in Rhizoglyphus mites; "fighter" males (top) use their modified 3rd pair of legs (arrow) to kill rival males but are less mobile than "scrambler" males (bottom). Ten generations of evolution in a complex environment shifted the underlying reaction norm, leading to a substantial decrease in the frequency of the fighter morph [224]. Copyright Jacek Radwan, Jagiellonian University, Kraków. (d) Populations of the guppy (Poecilia reticulata) introduced to sites free of the main predator (bottom) evolved brighter male coloration - here, blue dorsolateral spots and stronger blue-green iridescence on posterior body - compared to their ancestors (top) that evolved with visual predators [201]. Copyright Darrell J. Kemp, Macquarie University, Sydney. 